\title{
J. Herzog
}

Nagoya Math. J.

Vol. 83 (1981), 183-195

\section{WHEN IS A REGULAR SEQUENCE SUPER REGULAR?}

\section{J. HERZOG*)}

Let $(B, \mathscr{F})$ be a filtered, noetherian ring. A sequence $x=x_{1}, \cdots, x_{n}$ in $B$ is called super regular if the sequence of initial forms

$$
\xi_{1}=L\left(x_{1}\right), \cdots, \xi_{n}=L\left(x_{n}\right)
$$

is a regular sequence in $g r_{\mathscr{s}}(B)$.

If $B$ is local and the filtration $\mathscr{F}$ is $\mathfrak{A}$-adic then any super regular sequence is also regular, see [6], 2.4.

In [3], Prop. 6 Hironaka shows that in a local ring $(B, \mathfrak{M})$ an element $x \in \mathfrak{M} \mid \mathfrak{M}^{2}$ is super regular (with respect to the $\mathfrak{M}$-adic filtration) if and only if $x$ is regular in $B$ and $(x) \cap \mathfrak{M}^{n+1}=(x) \mathfrak{M}^{n}$ for every integer $n$.

This result is extended to a more general situation in [6], 1.1. In the present paper we will characterize super regular sequences in a relative case:

Let $A$ be a regular complete local ring, $B=A / I$ an epimorphic image of $A$ and $x=x_{1}, \cdots, x_{n}$ a regular sequence in $B$ which is part of a minimal system of generators of the maximal ideal of $B$. Let $y=y_{1}, \cdots, y_{n}$ be a sequence in $A$ which is mapped onto $x$. Then $y$ is part of a regular system of parameters of $A$. Therefore $y$ is a super regular sequence in $A$.

We put $\bar{A}=A /(y) A, \bar{I}=I /(y) I$ and $\bar{B}=B /(x) B$. Then $\bar{B}=\bar{A} / \bar{I}$, since $x$ is a $B$-sequence.

As a consequence of our main result, the following conditions are equivalent:

(a) $x$ is a super regular sequence in $B$

(b) For all elements $g \in \bar{I}$ there exists $f \in I$, such that

$$
\bar{f}=g \quad \text { and } \quad \nu(f)=\nu(g) .
$$

(Here $\bar{f}$ denotes the image of $f$ in $\bar{I}$ and $\nu(f)$ the degree of the initial form

Received October 6, 1979.

*) During the preparation of this work the author was supported by C.N.R. (Consiglio Nazionale delle Ricerche). 
of $f$.)

The equivalence of (a) and (b) can also be expressed in terms of Hironaka's numerical character $\nu^{*}(J, R): x$ is a super regular sequence in $B$ if and only if $\nu^{*}(I, A)=\nu^{*}(\bar{I}, \bar{A})$.

In the applications we will use this characterization to show that the tangent cone of certain algebras is CM (Cohen-Macaulay). Our examples contain some results of J. Sally [4], [5] in a more special case.

\section{§1. Notations and remarks}

In the following we fix our notations and recall some basic facts about filtrations. For a more detailed information about filtrations we refer to N. Bourbaki [1].

Let $(A, \mathscr{F})$ be a noetherian filtered ring such that $\mathscr{F}_{0} A=A$ and $\mathscr{F}_{i+1} A \subseteq \mathscr{F}_{i} A$ for $i \geq 0$ and let $(M, \mathscr{G})$ be a filtered $(A, \mathscr{F})$-module. Then $g r_{s}(M)=\oplus_{i \geq 0} \mathscr{G}_{i} M / \mathscr{G}_{i+1} M$ is a graded $g r_{s}(A)=\oplus_{i \geq 0} \mathscr{F}_{i} A / \mathscr{F}_{i+1} A$-module.

If $x \in M$ we define $\nu(x)=\sup \left\{n / x \in \mathscr{G}_{n} M\right\}$ to be the degree of $x$ and call

$$
L(x)=x+\mathscr{G}_{n+1} M \text { the initial form of } x .
$$

Let $\varphi: M \rightarrow N$ be a homomorphism of filtered modules then $\varphi$ induces a homogeneous homomorphism

$$
\operatorname{gr}(\varphi): \operatorname{gr}(M) \rightarrow \operatorname{gr}(N) .
$$

If $\varphi$ is an epimorphism, we always will assume that $N$ admits the canonical filtration induced from the filtration of $M$. Then

$$
\operatorname{Ker}(\operatorname{gr}(\varphi))=\{L(x) / x \in \operatorname{Ker} \varphi\} .
$$

We call a sequence $\left(x_{1}, \cdots, x_{n}\right), x_{i} \in \operatorname{Ker} \varphi$ a standard base of $\operatorname{Ker} \varphi$ if

$$
\operatorname{Ker}(\operatorname{gr}(\varphi))=\left(L\left(x_{1}\right), \cdots, L\left(x_{n}\right)\right) .
$$

In the particular case that $\varphi: A \rightarrow B$ is an epimorphism of filtered rings, we now give a slightly different but useful description of a standard base: Corresponding to a sequence $\left(x_{1}, \cdots, x_{n}\right), x_{i} \in \operatorname{Ker} \varphi$, we define a filtration on $A^{n}$ :

$$
\mathscr{F}_{i} A^{n}=\left\{\left(a_{1}, \cdots, a_{n}\right) / a_{j} \in \mathscr{F}_{i-\nu\left(x_{j}\right)} A\right\}
$$

Now 


$$
A^{n} \underset{\left(x_{1}, \cdots, x_{n}\right)}{\longrightarrow} A \underset{\varphi}{\longrightarrow} B \longrightarrow 0
$$

is a complex of filtered $A$-modules inducing a complex of $\operatorname{gr}(A)$-modules

$$
\operatorname{gr}\left(A^{n}\right) \underset{\left(L\left(x_{1}\right), \cdots, L\left(x_{n}\right)\right)}{\longrightarrow} \operatorname{gr}(A) \underset{\operatorname{gr}(\varphi)}{\longrightarrow} \operatorname{gr}(B) \longrightarrow 0
$$

and $\left(x_{1}, \cdots, x_{n}\right)$ is a standard base of $\operatorname{Ker} \varphi$ if and only if the complex (2) is exact.

If $A$ is complete and separated then any standard base of $\operatorname{Ker} \varphi$ is also a base of $\operatorname{Ker} \varphi$. However the converse is false in general.

Consider the following case:

Let $B=A / x A$, where $x$ is not a zero-divisor on $A$ and let $\varphi: A \rightarrow B$ be the canonical epimorphism and $\xi=L(x)$.

LEMMA. (a) If $x$ is super regular then

$$
\operatorname{gr}(A) \stackrel{\xi}{\longrightarrow} \operatorname{gr}(A) \stackrel{\operatorname{gr}(\varphi)}{\longrightarrow} \operatorname{gr}(B) \longrightarrow 0
$$

is exact, i.e. $(x)$ is a standard base of $\operatorname{Ker} \varphi=(x)$.

(b) If $A$ is complete and separated and the sequence (*) is exact then $x$ is super regular.

The lemma shows that a non-zero-divisor $x$ in a complete separated ring forms a standard base of $(x)$ if and only if it is super regular.

Proof of the lemma. (a) Let $\alpha \neq 0$ be a homogeneous element of $\operatorname{Ker}(\operatorname{gr}(\varphi))$. Then $\alpha=L(x a)$ for some $a \in A$. Since $\xi L(a) \neq 0$, we have $\xi L(a)=L(x a)=\alpha$.

(b) Let $\alpha \in \operatorname{gr}(A)$ be a homogeneous element such that $\xi \alpha=0$.

We construct a convergent series $\left(a_{n}\right)$ such that for all $n \geq 1$ we have $L\left(a_{n}\right)=\alpha$ and $\nu\left(x a_{n}\right) \geq \nu(x)+\nu\left(a_{1}\right)+n$.

Let $a=\lim a_{n}$, then $\alpha=L(a)$ and $x a \in \cap \mathscr{F}_{i} A=\{0\}$. Therefore $a=0$ and consequently $\alpha=0$. Construction of the sequence $\left(a_{n}\right)$ by induction on $n$ :

Let $a_{1} \in A$ such that $\alpha=L\left(a_{1}\right)$. Since $\xi \alpha=0$ we have $\nu\left(x a_{1}\right) \geq \nu(x)$ $+\nu\left(a_{1}\right)+1$.

Suppose we have already constructed $a_{1}, \cdots, a_{n}$. By induction hypothesis we have $\nu\left(x a_{n}\right) \geq \nu(x)+\nu\left(a_{1}\right)+n$. Since $L\left(x a_{n}\right) \in \operatorname{Ker}(\operatorname{gr}(\varphi))$ and since we suppose that $(*)$ is exact we find a homogeneous element $\gamma_{n}$ such that $\xi \gamma_{n}=L\left(x a_{n}\right)$. 
Choose $g_{n} \in A$ such that $\gamma_{n}=L\left(g_{n}\right)$, then $\nu\left(g_{n}\right)=\nu\left(x a_{n}\right)-\nu(x) \geq \nu\left(a_{1}\right)$ $+n$ and $\nu\left(x\left(a_{n}-g_{n}\right)\right) \geq \nu(x)+\nu\left(a_{1}\right)(n+1)$. The element $a_{n+1}=a_{n}-g_{n}$ is the next member of the sequence.

\section{§2. The main result}

Let $\varepsilon: A \rightarrow B$ be an epimorphism of complete and separated filtered rings. As before we assume that $B$ admits the induced filtration. Then Ker $\varepsilon$ is a closed ideal of $A$.

Suppose we are given a super regular sequence $y=y_{1}, \cdots, y_{n}$ on $A$ and let $x_{i}=\varepsilon\left(y_{i}\right)$. Suppose that $x=x_{1}, \cdots, x_{n}$ is a regular sequence on $B$ and that

$$
\nu\left(x_{i}\right)=\nu\left(y_{i}\right)>0
$$

for $i=1, \cdots, n$.

Let $\bar{A}=A /(y) A, \bar{B}=B /(x) B, I=\operatorname{Ker} \varepsilon$ and $\bar{I}=I /(y) I$. We have $\bar{I} \subset \bar{A}$ and $\bar{B}=\bar{A} / \bar{I}$, since $x$ is a regular sequence on $B$. If $f$ is an element of $A$ or of $B$ we denote its image in $\bar{A}$ or $\bar{B}$ by $\bar{f}$.

THEOREM 1. 1) The following properties are equivalent:

a) For each $g \in \bar{I}$ there exists $f \in I$ such that $\bar{f}=g$ and $\nu(f)=\nu(g)$.

b) There exists a standard base $g_{1}, \cdots, g_{m} \in \bar{I}$ and elements $f_{i} \in I$ such that $\bar{f}_{i}=g_{i}$ and $\nu\left(f_{i}\right)=\nu\left(g_{i}\right)$ for $i=1, \cdots, m$.

c) $x$ is a super regular sequence.

2) If the equivalent conditions of 1) hold and the $f_{i}$ are chosen as in b), then $\left(f_{1}, \cdots, f_{m}\right)$ is a standard base of $I$.

Proof. It is sufficient to consider the case that the sequence $x$ consists only of one element. The general case follows by induction on the length of the sequence.

1) a) $\Rightarrow$ b): is obvious

b) $\Rightarrow$ c): Let $\left(g_{1}, \cdots, g_{m}\right)$ be a standard base of $\bar{I}$ and $f_{i} \in I$ be such that $\bar{f}_{i}=g_{i}$ and $\nu\left(f_{i}\right)=\nu\left(g_{i}\right)$.

We define on $A^{n}$ and $\bar{A}^{n}$ filtrations

$$
\begin{aligned}
& \mathscr{F}_{i} A^{n}=\left\{\left(a_{1}, \cdots, a_{n}\right) / a_{j} \in \mathscr{F}_{i-\nu\left(f_{j}\right)} A\right\} \\
& \mathscr{F}_{i} \bar{A}^{n}=\left\{\left(\bar{a}_{1}, \cdots, \bar{a}_{n}\right) / \bar{a}_{j} \in \mathscr{F}_{i-\nu\left(g_{j}\right)} \bar{A}\right\}
\end{aligned}
$$

and obtain a commutative diagram of filtered modules 


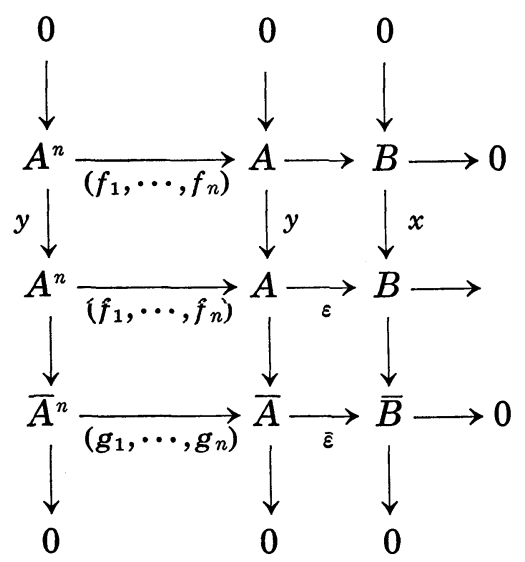

inducing a commutative diagram of graded modules

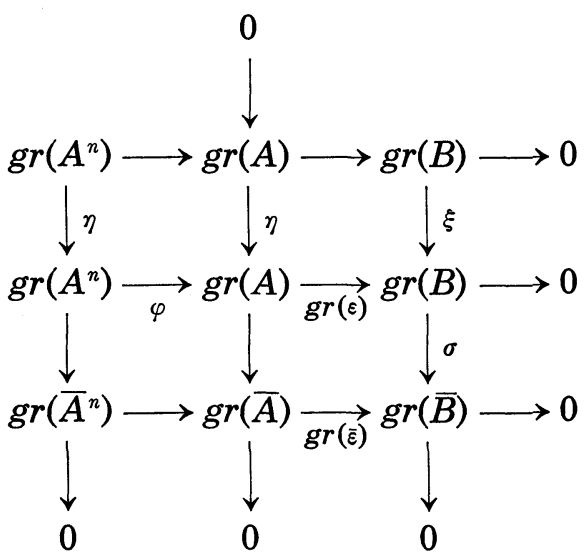

$\xi=L(x), \eta=L(y)$.

The lowest row is exact since $\left(g_{1}, \cdots, g_{n}\right)$ is a standard base. Also the middle column is exact since $y$ is super regular.

By diagram chasing we find, that also the sequence

$$
\operatorname{gr}(B) \stackrel{\xi}{\longrightarrow} \operatorname{gr}(B) \longrightarrow \operatorname{gr}(\bar{B}) \longrightarrow 0 \text { is exact. }
$$

By the lemma it follows that $x$ is super regular. c) $\Rightarrow$ a): Let $g \in \bar{I}$, then we can find an element $f \in A$ such that $\bar{f}=g$ and $\nu(f)=\nu(g)$.

However we would like to find such an element $f$ in $I$. To do this we consider

$$
\operatorname{\sigma gr}(\varepsilon)(L(f))=\operatorname{gr}(\bar{\varepsilon})(L(g))=0 .
$$


Since we assume that $x$ is super regular it follows from the lemma that $\operatorname{gr}(\varepsilon)(L(f))=\beta \xi$. Therefore $L(f)=\alpha \eta+\gamma$, where $\alpha, \gamma$ are homogeneous and $\gamma \in \operatorname{Ker}(\operatorname{gr}(\varepsilon))$.

Hence we can choose $a_{1}, f_{1} \in A$ and $h_{1} \in I$ such that

$$
\begin{aligned}
f & =a_{1} y+h_{1}+f_{1}, \\
\nu(f) & =\nu\left(a_{1} y\right)=\nu\left(h_{1}\right)<\nu\left(f_{1}\right) .
\end{aligned}
$$

From this we obtain $g=\bar{f}=\bar{h}_{1}+\bar{f}_{1} \in \bar{I}$, hence $\bar{f}_{1} \in \bar{I}$. Repeating the same reasoning for $f_{1}$, we can find $a_{2}, f_{2} \in A$ and $h_{2} \in I$ such that

$$
\begin{aligned}
f_{1} & =a_{2} y+h_{2}+f_{2}, \\
\nu\left(f_{1}\right) & =\nu\left(a_{2} y\right)=\nu\left(h_{2}\right)<\nu\left(f_{2}\right) .
\end{aligned}
$$

This time it may happen that $\nu\left(f_{1}\right)<\nu\left(\bar{f}_{1}\right)$, but that doesn't matter and we can take $h_{2}=0$ in that case. Proceeding that way we construct sequences $\left(a_{i}\right),\left(h_{i}\right)$ and $\left(f_{i}\right)$ such that $h_{i} \in I$ and

$$
\begin{aligned}
f_{i} & =a_{i} y+h_{i}+f_{i+1} \\
\nu\left(f_{i}\right) & =\nu\left(a_{i} y\right)=\nu\left(h_{i}\right)<\nu\left(f_{i+1}\right) .
\end{aligned}
$$

Put $a=\sum_{i=1}^{\infty} a_{i}, h=\sum_{i=1}^{\infty} h_{i}$. Then $f=a y+h, h \in I$ and $\nu(h)=\nu\left(h_{1}\right)=$ $\nu(f)=\nu(g)$. Thus $h$ is the desired element.

2) Consider again the diagram (2). We have to show that if $\alpha \epsilon$ $\operatorname{Ker}(\operatorname{gr}(\varepsilon))$ is a homogeneous element then there exists $\gamma \in \operatorname{gr}\left(A^{n}\right)$ such that $\varphi(\gamma)=\alpha$. We prove this by induction on the degree of $\alpha$. If $\operatorname{deg} \alpha<0$, then $\alpha=0$. Thus suppose that $\operatorname{deg} \alpha>0$. By assumption all columns and the lowest row are exact. By diagram chasing we can find homogeneous elements $\beta, \delta$ such that

$$
\alpha=\beta \eta+\delta,
$$

where $\delta \in \operatorname{Im} \varphi$ and $\beta \in \operatorname{Ker}(\operatorname{gr}(\varepsilon))$. Since by assumption $\operatorname{deg} \eta>0$, we have $\operatorname{deg} \beta<\operatorname{deg} \alpha$. From the induction hypothesis the assertion follows.

\section{§3. Some applications}

(a) Let $B=k\left[\left[x_{1}, \cdots, x_{n}\right]\right] / I$ be a 1-dimensional complete algebra over an algebraically closed field $k$. In the following we consider only the $m_{B^{-}}$ adic filtration of $B$.

Suppose that the residue class $x_{1}$ of $X_{1}$ is not a zero-divisor and a superficial element of $B$, then $\operatorname{gr}(B)$ is a CM-ring (Cohen-Macaulay) if 
and only if $x_{1}$ is super regular on $B$.

Applying Theorem 1 we find:

$\operatorname{gr}(B)$ is a CM-ring if and only if for all $F \in I$ there exists $G \in k\left[\left[X_{1}, \cdots, X_{n}\right]\right]$ such that

$$
F\left(0, X_{2}, \cdots, X_{n}\right)+G X_{1} \in I \text { and } \nu(G) \geq \nu\left(F\left(0, X_{2}, \cdots, X_{n}\right)\right)-1 .
$$

Next we restrict our attention to the more special case that $B$ is a monomial ring:

Let $H \subset N$ be a numerical semigroup generated minimally by $n_{1}<n_{2}<\ldots$ $<n_{l}$, see [2].

To $H$ belongs the monomial ring $B=k\left[t^{n_{1}}, \cdots, t^{n_{l}}\right]$, whose maximal ideal is $m_{B}=\left(t^{n_{1}}, \cdots, t^{n_{l}}\right)$. We want to describe in terms of the semigroup when $g r_{m_{B}}(B)$ is a CM-ring.

$t^{n_{1}}$ is a superficial element of $B$. Let $\bar{B}=B / t^{n_{1}} B \simeq k\left[\left[X_{2}, \cdots, X_{l}\right] / \bar{I}\right.$. It is easy to see that a standard base of $\bar{I}$ can be chosen such that the elements of the base are either monomials $X_{2}^{\nu_{2}} \cdots X_{l}^{\nu_{l}}$ or differences of monomials

$$
X_{2}^{\mu_{2}} \cdots X_{1}^{\mu_{1}}-X_{2}^{\mu_{2}^{*}} \cdots X_{l}^{\mu_{i}^{*}}
$$

with

$$
\sum_{i=2}^{l} \mu_{i} n_{i}=\sum_{i=2}^{l} \mu_{i}^{*} n_{i}
$$

Let $n_{1}+H=\left\{n_{1}+h / h \in H\right\} . \quad$ A monomial $X_{2}^{\nu_{2}}, \cdots, X_{l}^{\nu_{l}}$ is an element of $\bar{I}$ if and only if

$$
\sum_{i=2}^{l} \nu_{i} n_{i} \in n_{1}+H
$$

Thus we find:

$\operatorname{gr}(B)$ is a CM-ring if and only if for all integers $\nu_{2} \geq 0, \nu_{3} \geq 0, \cdots, \nu_{l} \geq 0$ such that

$$
\sum_{i=2}^{l} \nu_{i} n_{i} \in n_{1}+H
$$

there exist $\nu_{1}^{*}>0, \nu_{2}^{*} \geq 0, \cdots, \nu_{l}^{*} \geq 0$ such that

$$
\sum_{i=2}^{l} \nu_{i} n_{i}=\sum_{i=1}^{l} \nu_{i}^{*} n_{i} \quad \text { and } \quad \sum_{i=2}^{l} \nu_{i} \leq \sum_{i=1}^{1} \nu_{i}^{*}
$$

It is not difficult to see that it suffices to consider only such $\nu_{i}$ with 
the extra condition that $n_{i}>\nu_{i}$. Therefore only a finite number of conditions are to be checked.

If in addition $\bar{I}$ is generated only by monomials, then there is a unique minimal system of generators of $\bar{I}$ consisting of monomials $M_{1}, \cdots, M_{k}$. These monomials form a standard base of $\bar{I}$.

Thus $\operatorname{gr}(B)$ is a CM-ring if and only if to each such monomial

$$
M_{i}=X_{2}^{\nu_{2}} \cdots X_{l}^{\nu_{1}}
$$

we can find

$$
F_{i}=X_{2}^{\nu_{2}} \cdots X_{l}^{\nu_{l}}-X_{1}^{\nu_{1}^{*}} \cdots X_{l}^{\nu^{*}} \in I
$$

with

$$
\nu_{1}^{*}>0 \text { and } \sum_{i=2}^{l} \nu_{i} \leq \sum_{i=1}^{l} \nu_{i}^{*} .
$$

In particular if $\operatorname{gr}(B)$ is a CM-ring then $F_{1}, \cdots, F_{k}$ forms a standard base of $I$ and also a minimal base of $I$.

We now discuss in more detail monomial rings of embedding dimension 3. These examples were first studied by G. Valla and R. Robbiano in [7] and communicated to me, when I was visiting Genova. Using different methods they are able to construct in all cases a standard base. Here we restrict ourselves to the question whether $\operatorname{gr}(B)$ is a CM-ring.

Let $B=k\left[\left[t^{n_{1}}, t^{n_{2}}, t^{n_{3}}\right]\right]$ and assume first that $B$ is not a complete intersection. In [2] it is shown that $I=\left(F_{1}, F_{2}, F_{3}\right)$ with

$$
\begin{aligned}
& F_{1}=X_{1}^{c_{1}}-X_{2}^{r_{12}} \cdot X_{3}^{r_{13}} \\
& F_{2}=X_{2}^{c_{2}}-X_{1}^{r_{21}} \cdot X_{2}^{r_{23}} \\
& F_{3}=X_{3}^{c_{3}}-X_{1}^{r_{31}} \cdot X_{2}^{r_{32}}
\end{aligned}
$$

where $r_{i j}>0$ and $c_{1}=r_{21}+r_{31}, c_{2}=r_{12}+r_{32}$ and $c_{3}=r_{13}+r_{23}$. It follows that $\bar{I}$ is generated by monomials and therefore $\operatorname{gr}(B)$ is a CM-ring if and only if

$$
\begin{aligned}
& c_{1} \geq r_{12}+r_{13} \\
& c_{2} \leq r_{21}+r_{23} \\
& c_{3} \leq r_{31}+r_{32} .
\end{aligned}
$$

The first inequality is always satisfied since

$$
c_{1} n_{1}=r_{12} n_{2}+r_{13} n_{3}
$$


and

$$
n_{1}<n_{2}<n_{3}
$$

Similarly the third inequality is always true. Our final result is therefore: $\operatorname{gr}(B)$ is a CM-ring if and only if $c_{2} \leq r_{21}+r_{23}$.

\begin{tabular}{rrr||ccc|l}
$n_{1}$ & $n_{2}$ & $n_{3}$ & $c_{2}$ & $r_{21}$ & $r_{23}$ & CM \\
\hline 3 & 4 & 5 & 2 & 1 & 1 & Yes \\
5 & 6 & 13 & 3 & 1 & 1 & No
\end{tabular}

We now assume that $B=k\left[\left[t^{n_{1}}, t^{n_{2}}, t^{n_{3}}\right]\right]$ is a complete intersection. Then $I$ can be generated by two elements $F_{1}, F_{2}$. We have to distinguish several case:

\begin{tabular}{|c|c|c|}
\hline Case & $F_{1}, F_{2}$ & Example \\
\hline$\alpha)$ & $X_{1}^{c_{1}}-X_{2}^{c_{2}}, X_{1}^{c_{1}}-X_{3}^{c_{3}}$ & $6,10,15$ \\
\hline$\beta)$ & $X_{2}^{c_{2}}-X_{3}^{c_{3}}, X_{1}^{c_{1}}-X_{2}^{r_{12}} \cdot X_{3}^{r_{13}}$ & $7, \quad 9,12$ \\
\hline$\gamma)$ & $X_{1}^{c_{1}}-X_{3}^{c_{3}}, X_{2}^{c_{2}}-X_{1}^{r_{21}} \cdot X_{3}^{r_{23}}$ & $4, \quad 5,6$ \\
\hline$\delta)$ & $\begin{array}{l}X_{1}^{c_{1}}-X_{2}^{c_{2}}, X_{3}^{c_{3}}-X_{1}^{r_{31}} \cdot X_{2}^{r_{32}} \\
\quad \text { all } r_{i j}>0\end{array}$ & $4,6,7$ \\
\hline
\end{tabular}

Case $\alpha) . \quad \bar{I}=\left(X_{2}^{c_{2}}, X_{3}^{c_{3}}\right)$ is generated by monomials. Since $c_{1}>c_{2}$ and $c_{1}>c_{3}$, it follows that $B$ is a strict complete intersection.

Case $\beta) . \quad \bar{I}=\left(X_{2}^{c_{2}}-X_{3}^{c_{3}}, X_{2}^{r_{12}} \cdot X_{3}^{r_{13}}\right)$.

We want to find a standard base of $\bar{I}$ :

$$
X_{2}^{c_{2}+r_{12}}, X_{3}^{c_{3}}, X_{2}^{r_{12}} \cdot X_{3}^{r_{13}}
$$

are relations of $\operatorname{gr}(\bar{B})$. We easily compute the length $l$ of

$$
k\left[\left[X_{2}, X_{3}\right]\right] /\left(X_{2}^{c_{2}+r_{12}}, X_{3}^{c_{3}}, X_{2}^{r_{12}} \cdot X_{3}^{r_{13}}\right)
$$

to be

$$
l=r_{12} c_{3}+r_{13} c_{2}
$$

On the other hand we have

$$
n_{2}=c_{3} c_{1}, n_{3}=c_{2} c_{1}
$$

and

$$
c_{1} n_{1}=r_{12} n_{2}+r_{13} n_{3},
$$


therefore

$$
n_{1}=r_{12} c_{3}+r_{12} c_{2}=l \text {. }
$$

Since

$$
n_{1}=1\left(B / t^{n_{1}} B\right)=l\left(g r\left(B / t^{n_{1}} B\right)\right),
$$

it follows that

$$
X_{2}^{c_{2}+r_{12}}, X_{2}^{c_{2}}-X_{3}^{c_{3}}, X_{2}^{r_{12}} X_{3}^{r_{13}}
$$

is a standard base of $\bar{I}$.

There is only one way to lift these equations:

$$
X_{2}^{c_{2}+r_{12}}-X_{3}^{c_{3}-r_{13}} \cdot X_{1}^{c_{1}}, X_{2}^{c_{2}}-X_{3}^{c_{3}}, X_{1}^{c_{1}}-X_{2}^{r_{12}} \cdot X_{3}^{r_{13}} .
$$

Since $c_{1} \geq r_{12}+r_{13}$, we find that $\operatorname{gr}(B)$ is a CM-ring if and only if

$$
c_{2}+r_{12} \leq c_{3}-r_{13}+c_{1} \text {. }
$$

However $B$ is never a strict complete intersection.

r) $\bar{I}=\left(X_{3}^{c_{3}}, X_{2}^{c_{2}}\right)$ is generated by monomials. Thus $B$ is a strict complete intersection if and only if $c_{2} \leq r_{21}+r_{23}$.

ס) $\bar{I}=\left(X_{2}^{c_{2}}, X_{3}^{c_{3}}\right)$ is generated by monomials and $c_{3}<r_{31}+r_{32}$, therefore $B$ is always a strict complete intersection.

TheOREM 2. Let $B=k\left[\left[X_{1}, \cdots, X_{n}\right]\right] / I$ be a complete k-algebra and suppose that $I$ admits a standard base $F_{1}, \cdots, F_{m}$ such that:

1) $\nu\left(F_{i}\right)=2$ for $i=1, \cdots, m$.

2) For each homomorphism $\varphi: I / I^{2} \rightarrow B$ the elements $\varphi\left(F_{i}+I^{2}\right), i=$ $1, \cdots, m$ are not units in $B$ (equivalently, $B$ is not $a$ direct summand of $\left.I / I^{2}\right)$.

Then for any complete algebra $\tilde{B}=k\left[\left[Y_{1}, \cdots, Y_{k}\right]\right] / J$ and any regular $\tilde{B}-$ sequence $t_{1}, \cdots, t_{k}$ such that $\tilde{B} /\left(t_{1}, \cdots, t_{k}\right) \tilde{B}=B$ it follows that $\left(t_{1}, \cdots, t_{k}\right)$ is a super regular sequence on $\tilde{B}$.

Proof. We may write

$$
\tilde{B} \simeq k\left[\left[X_{1}, \cdots, X_{n}, T_{1}, \cdots, T_{k}\right]\right] / J
$$

such that $t_{i}=T_{i}+J, i=1, \cdots, k$. Then $J=\left(G_{1}, \cdots, G_{m}\right)$ with

$$
G_{i}=F_{i}+\sum_{j=1}^{k} F_{i}^{(j)} T_{j}+H_{i}
$$


$H_{i} \in\left(T_{1}, \cdots, T_{k}\right)^{2}$ and $F_{i}^{(j)} \in k\left[\left[X_{1}, \cdots, X_{n}\right]\right]$. Since $t_{1}, \cdots, t_{k}$ is a regular $\tilde{B}$ sequence, we obtain $B$-module homomorphisms

$$
\begin{aligned}
\varphi_{j}: & I / I^{2} \rightarrow B, \\
& F_{i}+I^{2} \mapsto F_{i}^{(j)}+I=1, \cdots, m
\end{aligned}
$$

By assumption 2) it follows that $\nu\left(F_{i}^{(j)}\right) \geq 1$ and by assumption 1) it follows that $\nu\left(G_{i}\right)=\nu\left(F_{i}\right)$ for $i=1, \cdots, m$.

From our criterion of section 2 the assertion follows.

We use this theorem to derive two results of J. Sally in a slightly more special case.

We introduce the following notations: $e(B)=$ embedding dimension of $B, d(B)=$ Krull dimension of $B$ and $m(B)=$ multiplicity of $B$.

THEOREM 3 ([4], [5]). Let $B \simeq k\left[\left[X_{1}, \cdots, X_{n}\right]\right] / I$ be a complete $C M$-algebra and suppose that either

a) $m(B) \leq e(B)-d(B)+1$

or

B) $m(B) \leq e(B)-d(B)+2$ and $B$ is a Gorenstein ring

then $\operatorname{gr}(B)$ is a CM-ring.

Proof. We may assume that $k$ is algebraically closed.

$\alpha)$ There exists a regular sequence $\left(t_{1}, \cdots, t_{d}\right)$ such that

$$
l\left(B /\left(t_{1}, \cdots, t_{d}\right) B\right)=m(B) .
$$

This sequence is part of a minimal system of generators of $m_{B}$. Let $\bar{B}=$ $B /\left(t_{1}, \cdots, t_{d}\right) B$, then $e(\bar{B})=e(B)-d(B)=m(B)-1=l(\bar{B})-1$. It follows that $m_{B}^{2}=0$, and $\bar{B}=k\left[\left[X_{1}, \cdots, X_{m}\right] / \bar{I}\right.$ with $\bar{I}=\left(X_{1}, \cdots, X_{m}\right)^{2}$. We may assume that $m \geq 2$ and show that $\bar{B}$ satisfies the conditions of Theorem 2 .

Condition 1 ) is obviously satisfied since $\bar{I}$ is generated by the monomials $X_{i} X_{j}$ of degree 2, which form a standard base of $\vec{I}$.

Suppose there exists a $\bar{B}$-module homomorphism $\varphi: \bar{I} / \bar{I}^{2} \rightarrow \bar{B}$ and integers $i, j$ such that $\varphi\left(X_{i} X_{j}+\bar{I}^{2}\right)$ is a unit.

1st Case. If $i=j$, then for any $k \neq i$ we have

$$
x_{k} \varphi\left(X_{i}^{2}+\bar{I}^{2}\right)+x_{i} \varphi\left(X_{i} X_{k}+\bar{I}^{2}\right),
$$

a contradiction since $\left(x_{1}, \cdots, x_{m}\right)$ is a minimal base of $m_{\bar{B}}$. 
2nd Case. If $i \neq j$, then $x_{i} \varphi\left(X_{i} X_{j}+\bar{I}^{2}\right)=x_{j} \varphi\left(X_{i}^{2}+\bar{I}^{2}\right)$, again a contradiction.

$\beta)$ As in the case $\alpha$ ) we can reduce $B$ to an algebra $\bar{B}$ such that $l(\bar{B})$ $=e(\bar{B})+2$. It follows that $m_{\bar{B}}^{3}=0$ and that $\bar{B}$ is a graded ring with Hilbert function $1+e(\bar{B}) t+t^{2}$. Let $\sigma$ be generator of $\bar{B}_{2}$. The multiplication on $\bar{B}$ induces a non singular quadratic form $\mathfrak{q}: \bar{B}_{1} \times \bar{B}_{1} \rightarrow k$ defined by

$$
\mathfrak{q}(v, w) \sigma=v \cdot w
$$

Since we assume that $k$ is algebraically closed we can choose a $k$ vectorspace base $x_{1}, \cdots, x_{m}$ of $\bar{B}_{1}$ such that $x_{i}^{2}=\sigma$ for $i=1, \cdots, m$ and $x_{i} x_{j}=0$ for $i \neq j$.

We treat the case $m=2$ separately, since in that case $\bar{B}$ is a complete intersection and Theorem 2 is not applicable. However then we have $B=k\left[\left[X_{1} \cdots X_{n}\right]\right] /\left(F_{1}, F_{2}\right)$ with $\bar{F}_{1}=X_{1}^{2}-X_{2}^{2}, \bar{F}_{2}=X_{1} X_{2}$. If $\nu\left(F_{1}\right)=$ $\nu\left(F_{2}\right)=2$, then the assertion follows from Theorem 1 . Otherwise, say $\nu\left(F_{1}\right)=1$, then $B$ is a hypersurface ring and the assertion follows again.

Now if $m>2$ we apply Theorem 2: Again the first condition is satisfied. We check condition 2):

1st Case. Suppose there exists a $\bar{B}$-module homomorphism $\varphi: \bar{I} / \bar{I}^{2} \rightarrow \bar{B}$ such that $\varphi\left(X_{1}^{2}-X_{i}^{2}+\bar{I}^{2}\right)$ is a unit, then

$$
\sigma \varphi\left(X_{1}^{2}-X_{i}^{2}+\bar{I}^{2}\right)=x_{1}^{2} \varphi\left(X_{1}^{2}-X_{i}^{2}+\bar{I}^{2}\right)=\varphi\left(X_{1}^{4}-X_{1}^{2} X_{i}^{2}+\bar{I}^{2}\right)=0,
$$

since $X_{1}^{4}-X_{1}^{2} X_{i}^{2} \in \bar{I}^{2}$. This is a contradiction.

2nd Case. Suppose there exists a $\bar{B}$-module homomorphism $\varphi: \bar{I} / \bar{I}^{2} \rightarrow$ $\bar{B}$ such that $\varphi\left(X_{i} X_{j}+I^{2}\right)$ is a unit, then $\sigma \varphi\left(X_{i} X_{j}+I^{2}\right)=x_{1}^{2} \varphi\left(X_{i} X_{j}+I^{2}\right)=$ $\varphi\left(\left(X_{1} X_{i}\right)\left(X_{1} X_{j}\right)+I^{2}\right)=0$ since $\left(X_{1} X_{i}\right)\left(X_{1} X_{j}\right) \in I^{2}$. This is again a contradiction.

\section{LITERATURE}

[1] N. Bourbaki, Algèbre commutative, Hermann, Fasc., XXVIIIf, Chap. 3.

[2] J. Herzog, Generators and Relations of Abelian Semigroups and Semigroup Rings, manuscripta math., 3 (1970), 175-193.

[ 3 ] H. Hironaka, Certain numerical characters of singularities, J. Math. Kyoto Univ., 10 (1970), 151-187.

[4] J. Sally, On the associated graded ring of a local Cohen-Macaulay ring, J. Math. Kyoto Univ., 17 (1977), 19-21.

[ 5 ] — - Tangent cones at Gorenstein singularities, to appear in Comp. Math.

[ 6 ] P. Vallabrega and G. Valla, Form rings and regular sequences, Nagoya Math. J., 72 (1978), 93-101. 
[ 7 ] G. Valla and L. Robbiano, On the equations defining tangent cones, to appear in Proc. Camb. Phil. Soc., 88 (1980), 281-297.

Universität Essen-Gesamthochschule

Fachbereich 6

Universitätsstr. 3 